\title{
An updated version of wannier90: A Tool for Obtaining Maximally-Localised Wannier Functions
}

\author{
Arash A. Mostofi ${ }^{\mathrm{a}, *}$, Jonathan R. Yates ${ }^{\mathrm{b}}$, Giovanni Pizzi ${ }^{\mathrm{f}}$, Young-Su Lee ${ }^{\mathrm{c}}$, \\ Ivo Souza $^{\mathrm{d}}$, David Vanderbilt ${ }^{\mathrm{e}}$, Nicola Marzarif ${ }^{\mathrm{f}}$ \\ ${ }^{a}$ Departments of Materials and Physics, Imperial College London, Exhibition Road, \\ London SW7 2AZ, UK \\ ${ }^{b}$ Department of Materials, University of Oxford, Parks Road, Oxford OX1 3PH, UK \\ ${ }^{c}$ High Temperature Energy Materials Research Center, Korea Institute of Science and \\ Technology, Seoul 136-791, Republic of Korea \\ ${ }^{d}$ Centro de Física de Materiales, Universidad del País Vasco, Paseo Manuel de \\ Lardizabal 5, 20018 San Sebastián, Spain \\ ${ }^{e}$ Department of Physics and Astronomy, Rutgers University, Piscataway, NJ \\ 08854-8019, USA \\ ${ }^{f}$ Theory and Simulation of Materials, École Polytechnique Fédérale de Lausanne, 1015 \\ Lausanne, Switzerland
}

\begin{abstract}
wannier90 is a program for calculating maximally-localised Wannier functions (MLWFs) from a set of Bloch energy bands that may or may not be attached to or mixed with other bands. The formalism works by minimising the total spread of the MLWFs in real space. This done in the space of unitary matrices that describe rotations of the Bloch bands at each $\mathrm{k}$ point. As a result, wannier90 is independent of the basis set used in the underlying calculation to obtain the Bloch states. Therefore, it may be interfaced straightforwardly to any electronic structure code. The locality of MLWFs can be exploited to compute band-structure, density of states and Fermi surfaces at modest computational cost. Furthermore, wannier90 is able to output MLWFs for visualisation and other post-processing purposes. Wannier functions are already used in a wide variety of applications. These include analysis of chemical bonding in real space; calculation of dielectric properties via the modern theory of polarisation; and as an accurate and
\end{abstract}

\footnotetext{
* Corresponding author.

E-mail address: a.mostofi@imperial.ac.uk
} 
minimal basis set in the construction of model Hamiltonians for large-scale systems, in linear-scaling quantum Monte Carlo calculations, and for efficient computation of material properties, such as the anomalous Hall coefficient. We present here an updated version of wannier90, wannier90 2.0, including minor bugfixes and parallel (MPI) execution for band-structure interpolation and the calculation of properties such as density of states, Berry curvature and orbital magnetisation. wannier90 is freely available under the GNU General Public License from http://www.wannier.org/.

Keywords: Electronic structure, density-functional theory, maximally-localized Wannier function PACS: 71.15.-m, 75.15.Ap, 73.22.-f, 31.10.+z

\section{NEW VERSION PROGRAM SUMMARY}

Manuscript Title: An updated version of wannier90: A Tool for Obtaining MaximallyLocalised Wannier Functions

Authors: Arash A. Mostofi, Jonathan R. Yates, Giovanni Pizzi, Young-Su Lee, Ivo Souza, David Vanderbilt and Nicola Marzari

Program Title: wannier90

Journal Reference:

Catalogue identifier:

Licensing provisions: This program is distributed under the GNU General Public License (see http://www.gnu.org/ for details)

Programming language: Fortran90, perl

Computer: any architecture with a Fortran 90 compiler Operating system: Linux, Windows, Solaris, AIX, Tru64 Unix, OSX

RAM: $10 \mathrm{Mb}$

Number of processors used: 1 or more

Keywords: Electronic structure, density-functional theory, Maximally-localized Wannier functions, band interpolation, transport, Berry phase

Classification: 7.3

External routines/libraries:

- BLAS (http://www/netlib.org/blas)

- LAPACK (http://www.netlib.org/lapack)

- MPI libraries (optional) for parallel execution

Catalogue identifier of previous version: aeak_v1_0

Journal reference of previous version: A. A. Mostofi, J. R. Yates, Y.-S. Lee, 
I. Souza, D. Vanderbilt and N. Marzari, "wannier90: A Tool for Obtaining Maximally-Localised Wannier Functions", Comput. Phys. Commun. 178, 685 (2008) [1]

Does the new version supersede the previous version?: Yes

Nature of problem:

Obtaining maximally-localised Wannier functions [2] from a set of Bloch energy bands that may or may not be entangled, and using these Wannier functions to calculate electronic properties of materials.

Solution method:

In the case of entangled bands, the optimally-connected subspace of interest is determined by minimising a functional which measures the subspace dispersion across the Brillouin zone. The maximally-localised Wannier functions within this subspace are obtained by subsequent minimisation of a functional that represents the total spread of the Wannier functions in real space. For the case of isolated energy bands only the second step of the procedure is required $[3,4]$.

Reasons for the new version:

Addition of new functionality, minor bug fixes, and parallel (MPI) execution for parts of the code.

Summary of revisions:

Enhancements include:

- Spinor projections

- Improved plotting

- Parallel execution

- Calculation of van der Waals interactions

- Landauer-Buttiker and Boltzmann transport

Full details are given in the CHANGE.log file, which can be found in the root directory of the distribution.

Running time:

Example calculations run in a few minutes.

[1] A. A. Mostofi, J. R. Yates, Y.-S. Lee, I. Souza, D. Vanderbilt and N. Marzari, "wannier90: A Tool for Obtaining Maximally-Localised Wannier Functions", Comput. Phys. Commun. 178, 685 (2008)

[2] N. Marzari, A. A. Mostofi, J. R. Yates, I. Souza and D. Vanderbilt, "MaximallyLocalised Wannier Functions: Theory and Applications", Rev. Mod. Phys. 84, 1419 (2012) 
[3] N. Marzari and D. Vanderbilt, "Maximally localized generalized Wannier functions for composite energy bands", Phys. Rev. B 56, 12847 (1997)

[4] I. Souza, N. Marzari and D. Vanderbilt, "Maximally localized Wannier functions for entangled energy bands", Phys. Rev. B 65, 035109 (2001) 